\title{
Statistical Properties of Bremsstrahlung Radiated by a Plasma
}

\author{
B. Crosignani, P. Di Porto \\ Fondazione U. Bordoni, Istituto Superiore P. T., Roma, Italy, \\ and F. Engelmann \\ Laboratori Gas Ionizzati (Associazione EURATOM-CNEN), Frascati, Italy \\ (Z. Naturforsch. 23 a, 968-969 [1968] ; received 25 March 1968)
}

\begin{abstract}
A formula is given for the correlation function of the bremsstrahlung emitted by a plasma, in terms of the correlations of the plasma density fluctuation. For the special case of stable and homogeneous plasmas in a steady state, the second-order correlation function is calculated as a functional of the particle distribution functions, and, as in the case of scattered laser light, found to have properties characteristic of a "chaotic" source. Generally, the possibility of gaining information about density correlations by photon counting experiments with bremsstrahlung turns out to be limited by the superposition of effects of many different Fourier components of the density fluctuations in the correlation functions of the radiation field.
\end{abstract}

The statistical properties of an electromagnetic field as investigated in the theory of optical coherence ${ }^{1,2}$ reflect directly the statistical structure of the source of the field. Along this line, some attention has been paid recently to the possibility of studying the statistical properties of a medium by investigating the statistics of photons scattered by it ${ }^{3-6}$. The problem has also been considered within the frame-work of very general assumptions on the dynamics of the source ${ }^{7}$. In this note we examine the case of bremsstrahlung radiated by a plasma.

The photon statistics at a point $\boldsymbol{R}$ is completely determined by the correlation functions of the electromagnetic field ${ }^{1,2}$. In the classical limit and for linearly polarized light, these are defined ${ }^{8}$ by the ensemble averages

$$
\begin{aligned}
& G^{(m)}\left(t_{1}^{\prime}, \ldots, t_{m}^{\prime}\right) \\
& =\left\langle\hat{E}^{*}\left(\boldsymbol{R}, t_{1}^{\prime}\right) \ldots \hat{E}^{*}\left(\boldsymbol{R}, t_{m}^{\prime}\right) \hat{E}\left(\boldsymbol{R}, t_{m}^{\prime}\right) \ldots \hat{E}\left(\boldsymbol{R}, t_{1}^{\prime}\right)\right\rangle \\
& \text { with } \quad \hat{E}(\boldsymbol{R}, t)=2 \int_{0}^{\infty} \mathrm{d} \omega E(\boldsymbol{R}, \omega) e^{i \omega t}
\end{aligned}
$$

the "analytic signal" 9 corresponding to the electric field

$$
E(\boldsymbol{R}, t)=\int_{-\infty}^{+\infty} \mathrm{d} \omega E(\boldsymbol{R}, \omega) e^{i \omega t}
$$

1 R. J. Glauber, Quantum Optics and Electronics, Les Houches 1964, edited by C. De Witt et al., Gordon and Breach Science Publisher Inc., New York 1965, p. 63.

2 L. Mandel and E. Wolf, Rev. Mod. Phys. 37, 231 [1965].

3 B. Crosignani and P. Di Porto, Phys. Letters 24 A, 69 [1967].

4 Y. R. Shen, Phys. Rev. 155, 921 [1967].

5 M. Bertolotti, B. Crosignani, P. Di Porto, and D. Sette, Phys. Rev. 157, 146 [1967].
For bremsstrahlung from a plasma consisting of electrons and (singly charged) ions, one has

$$
\begin{aligned}
E(\boldsymbol{R}, \omega)= & a \int_{-\infty}^{+\infty} \mathrm{d} \omega^{\prime} \int \mathrm{d}^{3} k^{\prime} n_{i}\left(\frac{\omega}{c} \boldsymbol{s}-\boldsymbol{k}^{\prime}, \omega-\omega^{\prime}\right) \\
& \cdot n_{\mathrm{e}}\left(\boldsymbol{k}^{\prime}, \omega^{\prime}\right) F\left(\boldsymbol{k}^{\prime}\right) e^{-i \omega R / c}
\end{aligned}
$$

with $n_{\mathrm{i}}(\boldsymbol{k}, \omega)$ and $n_{\mathrm{e}}(\boldsymbol{k}, \omega)$ the space-time Fourier transforms of the ion and electron density fluctuations, respectively, $a=2 e r_{0}^{2} /(2 \pi)^{4} R$ where $r_{0}$ is the classical electron radius, and

$$
F(\boldsymbol{k})=-\left(i / k^{2}\right)[(\boldsymbol{k} \times \boldsymbol{s}) \times \boldsymbol{s}] \cdot \boldsymbol{n}
$$

where $\boldsymbol{s}=\boldsymbol{R} / R$ and $\boldsymbol{n}$ denotes the unit vector in the direction of polarization considered. Eq. (2) follows readily by summing over the radiative parts of the electric fields ${ }^{10}$ around the single plasma electrons, accelerated in the Coulomb field of the ions and transforming to (fine-grained) densities. From Eqs. (1) and (2) it is seen that $G^{(m)}$ is related to correlations of the ion and electron density fluctuations of order $4 \mathrm{~m}$.

More explicit results require knowledge about the dynamics of the fluctuations of the plasma, i.e., a theory of plasma turbulence. Hence the problem

6 B. Crosignani, P. Di Porto, and F. Engelmann, Z. Naturforsch. 23 a, 743 [1968].

7 V. Korenman, Phys. Rev. 154, 1233 [1967].

8 For some more details cf. also ${ }^{6}$.

9 M. Born and E. Wolf, Principles of Optics, Pergamon Press Inc., New York 1964, p. 494.

10 See, e. g., K. H. Panofsky and M. Phillips, Classical Electricity and Magnetism, Addison-Wesley, London 1956. 
is, in the general case, fairly involved and only partial solutions exist. However, for stable plasmas with weak excitation of fluctuations, in which higher than binary correlations between particles are negligible, one obtains easily by "linearizing" the microscopic Vlasov equation ${ }^{8,11,12}$

$$
\begin{aligned}
n_{\mathrm{i}}(\boldsymbol{k}, \omega) & =D_{\mathrm{e}}(\boldsymbol{k}, \omega) n_{\mathrm{L}}^{(\mathrm{e})}(\boldsymbol{k}, \omega) \\
& +D_{\mathrm{i}}(\boldsymbol{k}, \omega) n_{\mathrm{L}}^{(\mathrm{i})}(\boldsymbol{k}, \omega), \\
n_{\mathrm{e}}(\boldsymbol{k}, \omega) & =B_{\mathrm{e}}(\boldsymbol{k}, \omega) n_{\mathrm{L}}^{(\mathrm{e})}(\boldsymbol{k}, \omega), \\
& +B_{\mathrm{i}}(\boldsymbol{k}, \omega) n_{\mathrm{L}}^{(\mathrm{i})}(\boldsymbol{k}, \omega),
\end{aligned}
$$

if the plasma is homogeneous and in a steady state. Here the quantities $n_{\mathrm{L}}^{(\mathrm{a})}(\boldsymbol{k}, \omega)$ with $\mathrm{a}=\mathrm{i}$, e are the space-time Fourier transforms of the "free" density fluctuations of ions and electrons, i. e.,

$$
\begin{aligned}
n_{\mathrm{L}}^{(\mathrm{a})}(\boldsymbol{k}, \omega)=\int \mathrm{d}^{3} \boldsymbol{r} \mathrm{d} t \exp \{i(\boldsymbol{k} \cdot \boldsymbol{r}-\omega t)\} \\
\cdot \sum_{j} \delta\left(\boldsymbol{r}-\boldsymbol{r}_{j}^{(\mathrm{a})}(0)-\boldsymbol{v}_{j}^{(\mathrm{a})}(0) t\right) \\
=2 \pi \sum_{j} \exp \left\{i \boldsymbol{k} \cdot \boldsymbol{r}_{j}^{(\mathrm{a})}(0)\right\} \delta\left(\omega-\boldsymbol{k} \cdot \boldsymbol{v}_{j}^{(\mathrm{a})}(0)\right)
\end{aligned}
$$

with $\boldsymbol{r}_{j}^{(\mathrm{a})}(0)$ and $\boldsymbol{v}_{j}^{(\mathrm{a})}(0)$ the position and velocity of the $j$-th particle of the species a at time $t=0$; the coefficients are defined by

$$
\begin{array}{ll}
B_{\mathrm{e}}=\frac{1-G_{\mathrm{i}}}{1-G_{\mathrm{e}}-G_{\mathrm{i}}}, & B_{\mathrm{i}}=\frac{-G_{\mathrm{e}}}{1-G_{\mathrm{e}}-G_{\mathrm{i}}}, \\
D_{\mathrm{e}}=\frac{-G_{\mathrm{i}}}{1-G_{\mathrm{e}}-G_{\mathrm{i}}}, & D_{\mathrm{i}}=\frac{1-G_{\mathrm{e}}}{1-G_{\mathrm{e}}-G_{\mathrm{i}}},
\end{array}
$$

where

$$
G_{\mathrm{a}}(\boldsymbol{k}, \omega)=\frac{\omega_{\mathrm{pa}}^{2}}{k^{2}} \lim _{\varepsilon \rightarrow 0} \int \mathrm{d}^{3} v \frac{\boldsymbol{k} \cdot \partial f^{(\mathrm{a})} / \partial \boldsymbol{v}}{\boldsymbol{k} \cdot \boldsymbol{v}-\omega+i \varepsilon},
$$

$f^{(\mathbf{a})}(\boldsymbol{v})$ and $\omega_{\mathrm{pa}}$ being the one-particle distribution function and the plasma frequency of the species a, respectively.

With the expressions of Eqs. (3) and (4) the correlation functions $G^{(m)}$ can be evaluated from Eqs. (1) and (2) by averaging over the initial values of the motion of the single particles. Since the plasma has been assumed stationary, their distribution is simply given by $f^{(\mathrm{a})}$. In particular, $G^{(1)}$ and $G^{(2)}$ result to be approximately given by

$$
\begin{aligned}
G^{(1)}\left(t_{1}{ }^{\prime}\right) \equiv\left\langle\hat{E}^{*}\left(\boldsymbol{R}, t_{1}{ }^{\prime}\right) \hat{E}\left(\boldsymbol{R}, t_{1}{ }^{\prime}\right)\right\rangle, & \\
G^{(2)}\left(t_{1}{ }^{\prime}, t_{2}{ }^{\prime}\right) & \equiv\left\langle\left|\hat{E}\left(\boldsymbol{R}, t_{1}{ }^{\prime}\right)\right|^{2}\right| \hat{E}\left(\boldsymbol{R}, t_{2}{ }^{\prime 2}\right\rangle \\
& =\left[G^{(1)}\left(t_{1}{ }^{\prime}\right)\right]^{2}+\left|\left\langle\hat{E}^{*}\left(\boldsymbol{R}, t_{1}{ }^{\prime}\right) \hat{E}\left(\boldsymbol{R}, t_{2}{ }^{\prime}\right)\right\rangle\right|^{2}
\end{aligned}
$$

$$
\begin{aligned}
\left\langle\hat{E}^{*}\left(\boldsymbol{R}, t_{1}{ }^{\prime}\right) E\left(\boldsymbol{R}, t_{2}{ }^{\prime}\right)\right\rangle=4 a^{2}(2 \pi)^{7} V \int \mathrm{d}^{3} v \mathrm{~d}^{3} v^{\prime} \mathrm{d}^{3} k|F(\boldsymbol{k})|^{2} Y\left[\boldsymbol{k} \cdot\left(\boldsymbol{v}-\boldsymbol{v}^{\prime}\right)\right] & (8) \\
\cdot & \cdot \exp \left\{i \boldsymbol{k} \cdot\left(\boldsymbol{v}-\boldsymbol{v}^{\prime}\right)\left(t_{1}^{\prime}-t_{2}^{\prime}\right)\right\}\left|D_{\mathrm{i}}(\boldsymbol{k}, \boldsymbol{k} \cdot \boldsymbol{v})\right|^{2} f^{(\mathrm{i})}(\boldsymbol{v})\left|B_{\mathrm{e}}\left(\boldsymbol{k}, \boldsymbol{k} \cdot \boldsymbol{v}^{\prime}\right)\right|^{2} f^{(\mathrm{e})}\left(\boldsymbol{v}^{\prime}\right),
\end{aligned}
$$

with

where $V$ is the plasma volume and $Y(\omega)=1$ for frequencies $\omega>0$ contributing to $\hat{E}(\boldsymbol{R}, t)$, but otherwise zero. Through the function $Y$ it is, hence, possible to take into account the effect of an arbitrary frequency filter and, in particular, the fact that the plasma does practically not emit frequencies below $\omega_{\text {pe }}$. In writing Eq. (7b) terms of the order $\lambda_{\mathrm{D}}{ }^{3} / V$ (with $\lambda_{\mathrm{D}}$ the Debye length) as well as low frequency contributions corresponding to $\omega \ll \omega_{\text {pe }}$ have been omitted. In Eq. (8) also terms of the order of the mass ratio $m_{\mathrm{e}} / m_{\mathrm{i}}$ are suppressed, assuming that the ion temperature does not appreciably exceed the electron temperature. Furthermore, relativistic effects, connected with the appearance of $\omega / c$ in the argument of $n_{\mathrm{i}}$ in Eq. (2), have been consistently neglected.

We emphasize that the relation (7b) for $G^{(2)}$, which also holds for laser light scattered by a stable plasma (cf. ${ }^{6}$ ), corresponds to what has been

11 Yu. L. Kumontovich, The Statistical Theory of Non-Equilibrium Processes in a Plasma, Pergamon Press, Inc. Oxford 1967 . obtained for a "chaotic" source (cf. $\left.{ }^{1}\right)$ : for $t_{2}{ }^{\prime}-t_{1}{ }^{\prime}$ $\rightarrow 0$ it is consistent with a Bose-Einstein statistics, for $t_{2}{ }^{\prime}-t_{1}{ }^{\prime} \rightarrow \infty$ with a Poisson statistics of the photons. Whereas in the case of scattered light the time characteristic of the transition from the first to the second regime is connected with the damping time of the relevant fluctuations, it can be shown from Eq. (8) that in the case of bremsstrahlung this time is of the order of the inverse of the bandwidth of the radiation received, independent of the frequency range considered. This result is due to the triple integration in (8) over $\boldsymbol{v}, \boldsymbol{v}^{\prime}$, and $\boldsymbol{k}$, which "smears out" the characteristics of single Fourier components of the fluctuations and shows an important general disadvantage of photon counting experiments with bremsstrahlung: they yield only global information about many (spatial) Fourier components of the density fluctuations, in contrast to light scattering experiments which single out just one of them.

12 I. Fidone, S. Lafleur, and Ch. Lafleur, Report EUR-CEAFC, $N^{\circ} 204$, Fontenay-aux-Roses (Hauts-de-Seine), France 1963. 